\title{
Joint Statement on new opportunities for air quality sensing - lower-cost sensors for public authorities and citizen science initiatives
}

\author{
Sven Schade ${ }^{\ddagger}$, Wiebke Herding ${ }^{\S}$, Arne Fellermannl, Alexander Kotsev ${ }^{\ddagger}$ \\ ‡ European Commission - Joint Research Centre, Ispra, Italy \\ $\S \mathrm{ON}:$ SUBJECT, Haarlem, Netherlands \\ | Bund für Umwelt und Naturschutz Deutschland e.V. (BUND), Berlin, Germany
}

Corresponding author: Sven Schade (s.schade@ec.europa.eu)

Reviewable v1

Received: 22 Feb 2019 | Published: 05 Jun 2019

Citation: Schade S, Herding W, Fellermann A, Kotsev A (2019) Joint Statement on new opportunities for air quality sensing - lower-cost sensors for public authorities and citizen science initiatives. Research Ideas and Outcomes 5 : e34059. https://doi.org/10.3897/rio.5.e34059

\begin{abstract}
Low-cost air quality sensors continue to spread. While their measurement quality does not compete with high-end instrumentation deployed in official air quality monitoring stations, they have a great potential to complement existing air quality assessments. However, we still see challenges related to data quality, data interoperability, and for collaborating on data assimilation and calibration. In order to move ahead we gathered as a group of 38 organisations from 14 different countries, including governmental authorities, network operators, citizen science initiatives, environmental Non-Governmental Organisations (NGOs), and academic researchers to explore how we can collaborate and better leverage each other's work. This statement captures our joint findings and recommendations.
\end{abstract}

\section{Our key observations include:}

- Co-operation between official monitoring networks (reference quality data) and lower-cost sensor operators is a key to make air quality data more usable.

- To be able to combine forces and benefit from each other's expertise, the different perspectives of all stakeholders should be taken into account. 
- There is a need to ensure that all users understand the possibilities and the limitations of making sense out of observations from different sensors.

- It is not realistic to expect that in the near future the data quality of lower-cost sensors will be as good as that of the official data. A way to make use of data that is of lower accuracy is by employing them in air quality modelling.

- Transparency about data quality is important to build more trust in the data, and to avoid unrealistic expectations.

- $\quad$ The need for interoperability should be clearly articulated and promoted by potential data users.

- $\quad$ There a need (and an opportunity) to provide guidance and standard operating procedures for the deployment and calibration of lower-cost sensors in order to increase the data quality delivered by participants of citizen science projects.

- $\quad$ Presently, we prefer to consider fixed-stationary sensors in a network instead of mobile sensor data. Furthermore, stationary data should not be aggregated with data from mobile sensors.

- $\quad$ Publishing and sharing this statement is only small step in the right direction and further actions have to be taken, inlcuding more in-depth discussions of the recommendations in smaller groups and follow-up meetings on dedicated topics.

\section{Keywords}

air quality, air pollution, low-cost sensing, citizen science, expert, stakeholders, joint statement

\section{Introduction}

Low-cost air quality sensors continue to spread. While their measurement quality does not compete with high-end instrumentation deployed in official air quality monitoring stations, they have a great potential to complement air quality assessments (Castell et al. 2017). From a scientific point of view sensors that are of lower-cost than those used within official stations may help us to create a spatially much denser sensing network and ultimately to improve our knowledge about air pollution. From a societal point of view the use of lowercost sensors may increase awareness, empower people, and have political impact.

Already today we witness:

- Sensors operated by citizens directly from homes, schools or other locations of interest (Grothe et al. 2016, Pfeil 2015).

- Initiatives promoting the use of lower-cost sensors organised by grass-root citizen communities, by academic research institutes, by environmental NGOs, by public authorities on the local, national or European level, or by consortia of these (Piedrahita et al. 2014). 
- A broadly felt need for data quality assessment, quality control, validation and calibration (Lewis et al. 2018).

- $\quad$ Rapid innovations in sensing technologies, including new fast on-the-fly methods for data quality assessment, quality control, validation and calibration (Maag et al. 2018, Piedrahita et al. 2014).

- $\quad$ Success stories of collaborations between public authorities and different kinds of lower-cost sensing initiatives (Volten et al. 2018).

On the one hand, we see improved data availability from both governmental air quality sensor networks and lower-cost sensing initiatives. But, what should be the next step in terms of interoperability and collaboration on data assimilation and calibration? How to ensure that different users understand the possibilities and the limitations of using observations from different sensors?

On the other hand, some agencies running official monitoring networks still seem to struggle to communicate their work and to reach public awareness. How could both citizen initiatives and public authorities benefit from each other's interests and mandates? How do we make sense of the air quality data we have available and communicate with citizens?

On the 24th of October 2018, we gathered as a group of governmental authorities, network operators, citizen science initiatives, environmental Non-Governmental Organisations (NGOs), and academic researchers to explore how we can collaborate and better leverage each other's work. All are listed in the Acknoweldgemet Section, below, and the meeting material is publicly available (http://www.hackair.eu/round-table-review/). In particular, we focus on three key questions, related with (i) the increased usability of lower-cost air quality data, (ii) combined use of data from heterogeneous sources, and (iii) our joint way forward. This statement captures our joint findings and recommendations.

\section{Increasing uptake of air quality from lower-cost sensing systems information in policy and society}

Sources of air quality information keep increasing while policy still seems to largely build on authoritative measurements. To use the potential of new sources of air quality data it would help if citizens, academics and policy makers interested in accessing (and contributing to) better air quality information from multiple sources would work together to increase the usefulness of the data, so that all parties may benefit.

To be able to combine forces and benefit from each other's expertise, the different perspectives of all stakeholders should be taken into account. We particularly want to underline the following reasons why different stakeholder groups should collaborate closely in order to increase the uptake of air quality from lower-cost sensing systems information in policy and society.

Reasons to involve citizens and bottom up-initiatives: 
- $\quad$ Benefit from existing enthusiasm, leaders and multipliers, and empower them with tools.

- $\quad$ Bring communities physically together and allow them to learn from one another.

- Make it easy to share technical knowledge and access existing solutions.

- Emphasise the context of the air quality data. What do limit values mean? What are the health implications?

Reasons to involve scientists and researchers:

- $\quad$ Take time at the beginning of a new initiative to clarify the research questions and methodology.

- Help translate scientific data into clear and simple communication (visuals, not just $\mu \mathrm{g} / \mathrm{ppm})$.

- Identifying the price gap between citizen science and authoritative research, the cost for calibration/scientific accuracy/maintenance is much higher than just sensors.

Reasons to involve public authorities and governmental agencies:

- Find possibilities for the uptake of air quality measurements from lower-cost sensors by public authorities.

- Identify possible conditions for data use, such as the requirement of clear information about the quality of the measurements and their source (metadata).

- Encouragement and support to engage multiple-actors and to use data from different sources.

Overall, we see a strong need for expectation management between all stakeholders involved. During the entire collaboration, it has to be made very clear what each party can and cannot offer.

\section{Making use of air quality data from multiple sources}

Making use of air quality data from multiple sources poses many scientific and technical challenges. We are looking for sound practical solutions that fit the needs of all stakeholders involved. From our joint discussions we conclude the following.

\section{Data quality: coping with varying levels of data quality}

1. We see a need (and an opportunity) to provide guidance and standard operating procedures for the deployment and calibration of lower-cost sensors in order to increase the data quality delivered by participants of citizen science projects. These guidance and procedures need to be developed by citizen scientists together with universities or research centres, and governmental agencies in order to satisfy the needs of all involved. 
2. One way to increase confidence and usability of lower-cost sensor data for science and policy is to complement sensor datasets with metadata describing data quality, e.g. (measurement accuracy, calibration, methodology used, etc.) following an agreed framework.

3. Stakeholders should be informed that sensor systems may under or overestimate the actual air pollutant levels. Because of the increasing volumes of sensor big data, new automatic procedures should be designed to detect data anomalies without human intervention.

4. For practical deployment by users it is important to estimate the lifetime of sensor systems and their periodicity of re-calibration.

5. One does not necessarily need data of the highest accuracy, but data of an accuracy that is known, and sufficient to address a given research question. For example, relative comparability of the data provided by a given (lower-cost) sensor system can be evaluated in order to ensure that sensors are able to capture the true spatial and temporal variability of air pollutants.

6. In order to make more sense of the data, sensor measurements may be compared and combined with data from other sources, including reference stations, meteorological conditions, models, and emission data.

7. At this point in time, we prefer to consider fixed-stationary sensors in a network instead of mobile sensor data. Furthermore, stationary data should not be aggregated with data from mobile sensors.

\section{Interoperability: comparing and integrating measurements from multiple sources}

1. The need for interoperability should be clearly articulated and promoted by potential data users (such as scientific institutions, and governmental organizations at different administrative levels), including aspects such as enabling data comparison and integration across borders (e.g. EU wide), easy access to a large data pool to directly work with, creating a unified market for entrepreneurs to build upon, making data relevant and accessible for policy, etc.

2. The re-use of data, sensing solutions and data management platforms should be rewarded by funding bodies.

3. Interoperability needs an adequate infrastructure that would facilitate the re-use and long-term curation of air sensing results. We welcome the developments of the European Open Science Cloud (EOSC) that might meet the demand of the community.

4. A comprehensive pool of supporting tools, made available under an open license would complement the emerging infrastructure for interoperable observation data.

5. The comparability and usefulness of data from lower-cost sensor systems may increase by developing, agreeing on and using standards and guidelines. At the same time we agree that no single solution can be adopted to meet all expectations. That is why, a brokering system that allows different already existing systems to interoperate could be put in place. 


\section{Modelling: analysing and learning from the multitude of air quality measurements available}

1. It is not realistic to expect that in the near future the data quality of lower-cost sensors will be as good as that of the official data. A way to make use of data that is of lower accuracy is by employing them in air quality modelling. Lower-cost sensor data may be used as input in models or to compare with models. Requirements for the data are not high accuracy, but to know the accuracy. This makes it a good first step in employing sensor for monitoring applications.

2. To be able to use the data it is essential to know which sensors are operating and which are not working. Operators should keep track of sensor up-time and send warnings to sensor owners in case of data lacks or atypical sensor behaviour. Air pollution models are already existing at EU-level. These can directly be used to investigate possible validation and modifications based on data from other sources, incl. lower-cost sensors systems. The output of CAMS (Copernicus Atmosphere Monitoring Service) can be used as a first guess.

3. One of the added benefits can be that the use of sensors and combination with modelling can increase the trust in models - which is a persistent issue. This work can open a dialogue and help increase the understanding of air quality models and their value.

4. Following the point above, model owners would benefit, because they can improve their models. Projects running lower-cost sensor systems would benefit because they cannot only present measurements at the points where there are systems deployed but also in the entire area in between. In addition, with models you can make air quality predictions.

\section{Calibration: improving the quality of air quality information}

1. Co-operation between official monitoring networks (reference quality data) and lower-cost sensor operators is a key to make air quality data more usable. This could, for example, include the offering of standards and certification mechanisms to communities that use lower-cost sensor systems.

2. Transparency about data quality is important to build more trust in the data, and to avoid unrealistic expectations.

3. A calibration/quality check is recommended, especially relating to calibration validity and representatives. This has to clarify the measurement range, meteorological condition of the sensor operation, environmental conditions, aerosol composition, etc.

4. Promote open access for comparison measurements.

5. When dealing with lower-cost sensor networks, projects should prominently and explicitly state what can be done with the data or by explaining what ancillary data is needed to make the data useful. Such a more positive framing would be preferred to any form of disclaimer about the data quality and validity which might discourage participation. A template of such statements might be shared to reduce the burden and guide projects. 
6. The aging of sensors and their need for maintenance should be studied and communicated to users.

\section{Data management: sharing data and honouring sources, confidentiality and ownership}

1. We identified a need to develop a data sharing framework that holds to legal requirements (esp. GDPR), explains possibilities on how to best preserve the privacy of participants, guides us to be (technically) interoperable, e.g. to approaches of public administration, and is fit for the future. Foresight is needed in order to address issues that might appear when sensors are carried by people including minors.

2. We suggest a dedicated meeting and surrounding activity in order to start building such a framework. Here, we see a need to invite researchers dealing with the ethics of technology, lawyers, data scientists, experts on interoperability.

3. For the short term we recommend to provide a guidebook (which might evolve incrementally) for new projects that are starting now. This should basically ask the question "What would we recommend these projects to do?".

4. Dedicated action is needed to investigate storage possibilities of citizen science data. Such an activity should also elaborate on the roles that citizens might play in this e.g. considering highly decentralised storage and involving the 'hacker' community.

\section{Moving ahead}

Given that this statement emerged from a single day meeting, we do see a need to take further action. In a nutshell, we consider the following actions most important and urgent to take:

- $\quad$ Sharing the results with the wider community and ensuring the re-use of existing practices, e.g. via the ECSA (European Citizen Science Association) Working Group on Air Quality, but also within the European Network of Air Quality Reference Laboratories (AQUILA).

- $\quad$ Collecting outcomes from research projects which show interdisciplinary results on the cross-cutting themes discussed in this Joint Statement.

- Keeping networked organizations aligned or forming a coordinated effort by relevant (country-based) bodies of institutions to keep an overview and up-to-date policy diagnosis of the learning curve that is currently taking shape.

- Facilitating exchange and dissemination of (inter-) disciplinary knowledge by the various actions and research projects that are being organized and rolled-out, both by local governments, research groups, civil groups and citizen science communities. 
- Taking up some of the recommendations of this document in smaller groups, including the organisation of a series of follow-up meetings on dedicated topics, elaborating further on the feedback received in Frankfurt.

\section{Acknowledgements}

This statement is a result of a meeting at which individual representatives of the following organizations took part:

$3 S \mathrm{GmbH}$ - Sensors, Signal Processing, Systems, Germany; AirBG.info, Bulgaria; Airthings, Bulgaria; Barcelona Institute for Global Health, Spain; Belgian Interregional Environment Agency (IRCELINE), Belgium; Breeze Technologies UG, Germany; BUND / Friends of the Earth, Germany; City of Oslo, Norway; DRAXIS Environmental S.A., Greece; Environmental social science research group, DIY Science $L A B$, Hungary; European Citizen Science Association (ECSA), Germany/UK; European Climate Foundation, UK; European Commission, DG Environment, Belgium; European Commission, DG Joint Research Centre, Italy; European Environment Agency(EEA), Denmark; Federal Environment Agency (Umweltbundesamt), Germany; Flanders Environment Agency (VMM), Belgium; Hessisches Landesamt für Naturschutz, Umwelt und Geologie, Germany; Institut für Geoinformatik, University of Münster, Germany; Institute for Advanced Architecture of Catalonia (IAAC)/Fablab Barcelona, Spain; Institute of Environmental Assessment and Water Research (IDAEA), Spain; Johannes GutenbergUniversity, Germany; Lambeth Borough Council, UK; Landesanstalt für Umwelt BadenWürttemberg, Germany; Leibniz Institute for Tropospheric Research (TROPOS), Germany; Leiden University, Netherlands; Luftdaten.info, Germany; Meritum, Poland; NanoSen-AQM, Spain; New Bulgarian University, Bulgaria; NILU - Norwegian Institute for Air Research, Norway; ON:SUBJECT, Belgium; Plume Labs, France; Radboud University, Netherlands; RIVM - National Institute of Public Health and the Environment, Netherlands; Sofia EU Green Capital, Bulgaria; Sofia Municipality, Bulgaria; Waag Society, Netherlands; and ZSI Centre for Social Innovation, Austria.

We particularly thank the speakers, the moderators of the different discussion groups at the Frankfurt workshop. In addition, we very much value the constructive and detailed comments of Wolfram Birmilli, Joost Wesseling and Hester Volten which helped to improve this joint statement.

The content of this publication does not reflect the official opinion of the European Union. Responsibility for the information and views expressed in the statement lies entirely with the authors. 


\section{Funding program}

European Union Horizon 2020 research and innovation programme, namely the hackAIR project (grant agreement no 688363) and the institutional financing of the Joint Research Centre (JRC) in the year 2018.

\section{Author contributions}

The authors of this article were the main organizers and facilitators of the round table that initiated this work and that generated to main inputs for this statement. They also edited the document at hand. All additional contributing organizations are listed in the Acknowledgements section. Contributions included presentations, moderation of group work, and active participation in the discussions during the meeting, as well as, contributions and agreements on the final statement.

\section{References}

- $\quad$ Castell N, Dauge F, Schneider P, Vogt M, Lerner U, Fishbain B, Broday D, Bartonova A (2017) Can commercial low-cost sensor platforms contribute to air quality monitoring and exposure estimates? Environment International 99: 293-302. https://doi.org/10.1016/ j.envint.2016.12.007

- $\quad$ Grothe M, Broecke JV, Carton LJ, Volten H, Kieboom R (2016) Smart Emission - Building a Spatial Data Infrastructure for an Environmental Citizen Sensor Network. In: Jirka S, Stasch C, Hitchcock A (Eds) Proceedings of the Geospatial Sensor Webs Conference 2016 (GSW 2016). Publisher, CEUR [ISBN SN16130073].

- Lewis AC, von Schneidemesser E, Peltier R (Eds) (2018) Low-cost sensors for the measurement of atmospheric composition: overview of topic and future applications. World Metrologic Organisation (WMO)

- Maag B, Zhou Z, Thiele L (2018) A Survey on Sensor Calibration in Air Pollution Monitoring Deployments. IEEE Internet of Things Journal 5 (6): 4857-4870. https://doi.org/10.1109/ jiot.2018.2853660

- $\quad$ Pfeil MBTWJA (2015) OPENSENSEMAP - A Citizen Science Platform For Publishing And Exploring Sensor Data as Open Data. University of Massachusetts Amherst https:// doi.org/10.7275/R56971SW

- $\quad$ Piedrahita R, Xiang Y, Masson N, Ortega J, Collier A, Jiang Y, Li K, Dick R, Lv Q, Hannigan $M$, Shang $L$ (2014) The next generation of low-cost personal air quality sensors for quantitative exposure monitoring. Atmospheric Measurement Techniques Discussions 7 (3): 2425-2457. https://doi.org/10.5194/amtd-7-2425-2014

- Volten H, Devilee J, Apituley A, Carton L, Grothe M, Keller CU, Kresin F, Land-Zandstra AM, Noordijk E, Putten Ev, Rietjens JH, Snik F, Tielemans E, Vonk J, Voogt M, Wesseling J (2018) Enhancing national environmental monitoring through local citizen science. In: Hecker S, Haklay M, Bowser A, Makuch Z, Vogel J, Bonn A (Eds) Citizen Science: Innovation in Open Science, Society and Policy. UCL Press [ISBN 978-1-78735-233-9]. 